\title{
Integrated optimisation framework for the design and scheduling of batch cooling water networks
}

\author{
Victor Dippenaar and Thokozani Majozi* \\ School of Chemical and Metallurgical Engineering, University of the Witwatersrand, 1 Jan \\ Smuts Avenue, Braamfontein, Johannesburg, 2000, South Africa \\ E-mail: thokozani.majozi@wits.ac.za \\ Phone: $+27(0) 117177384$
}

\section{Appendix A}

\section{Batch scheduling constraints}

\section{Assignment constraints}

The assignment constraints below dictate the assignment of tasks to unit operations. Constraints (1) and (2) ensure that a maximum of one task can start or finish in a unit $j$ at any time point $p$.

$$
\begin{array}{ll}
\sum_{i \in I_{j}(j)} W_{s}(i, p) \leq 1 & \forall j \in J, p \in P \\
\sum_{i \in I_{j}(j)} W_{f}(i, p) \leq 1 & \forall j \in J, p \in P
\end{array}
$$


Constraint (3) stipulates that all tasks that start must also finish.

$$
\sum_{p \in P} W_{s}(i, p)=\sum_{p \in P} W_{f}(i, p) \quad \forall i \in I
$$

Constraint (4) ensures that at most one task can be active in any unit operation $j$ at any time point $p$.

$$
\sum_{i \in I_{j}(j)} \sum_{p^{\prime} \in P}^{p^{\prime} \leq p}\left(W_{s}\left(i, p^{\prime}\right)-W_{f}(i, p)\right) \leq 1 \quad \forall j \in J, p \in P
$$

The conditions that tasks cannot finish at the first time point or start at the final time point are enforced by constraints (5) and (6) respectively.

$$
\begin{array}{cl}
W_{f}(i, p)=0 & \forall i \in I, p \in P, p=p_{1} \\
W_{s}(i, p)=0 & \forall i \in I, p \in P, p=|P|
\end{array}
$$

\section{Duration and sequencing constraints}

The duration and sequencing constraints below are concerned with task duration, the time horizon and the location of the time points. Constraints (7) and (8) ensure that the first time point occurs at the start of the time horizon and the final time point occurs at the end of the time horizon, respectively.

$$
\begin{gathered}
t(p)=0 \quad \forall p \in P, p=p_{1} \\
t(p)=H \quad \forall p \in P, p=|P|
\end{gathered}
$$

Constraint (9) states that the time at which each time point takes place must be greater than or equal to the time at which the previous time point took place.

$$
t(p+1) \geq t(p) \quad \forall p \in P, p \neq|P|
$$


The duration constraint is depicted by constraint (10). It is worth mentioning that the duration of each task is made up of a constant processing term, depending on whether the task is active, and a variable processing term, depending on batch size.

$$
\tau(i, p)=\alpha(i) W_{s}(i, p)+\beta(i) B_{s}(i, p) \quad \forall i \in I, p \in P
$$

The finish time of a task at a particular time point is expressed by making use of big-M constraints. These constraints are active only if the particular task $i$ starts at time point $p$. This can be seen from constraints (11) and (12).

$$
\begin{array}{ll}
t_{f}(i, p) \leq t_{s}(i, p)+\tau(i, p)+H\left(1-W_{s}(i, p)\right) & \forall i \in I, p \in P \\
t_{f}(i, p) \geq t_{s}(i, p)+\tau(i, p)-H\left(1-W_{s}(i, p)\right) & \forall i \in I, p \in P
\end{array}
$$

Constraint (13) uses the big-M formulation to ensure that the finish time of a particular task $i$ at time point $p$ remains unchanged until the next occurrence of task $i$.

$$
t_{f}(i, p)-t_{f}(i, p-1) \leq H \cdot W_{s}(i, p) \quad \forall i \in I, p \in, p>p_{1}
$$

Constraints (14) and (15) stipulate that the finish time of a task $i$ at time point $p$ is greater or equal to the finish time of task $i$ at the previous time point. Should the particular task $i$ take place at time point $p$, these constraints ensure that the difference in finishing times for the successive time points should at least be equal to the task duration.

$$
\begin{gathered}
t_{f}(i, p)-t_{f}(i, p-1) \geq \tau(i, p) \quad \forall i \in I, p \in P, p>p_{1} \\
t_{f}(i, p) \geq \tau(i, p) \quad \forall i \in I, p \in P, p=p_{1}
\end{gathered}
$$


Constraint (16) coordinates the time at which time point $p$ occurs to coincide with the start time of any task $i$ at time point $p$.

$$
t_{s}(i, p)=t(p) \quad \forall i \in I, p \in P
$$

Constraints (17) and (18) are known as the time-matching constraints for the finishing time of a task. The general equation allows for a task $i$ to finish at or before a time point $p$. However, if the task requires cooling water or produces a zero wait state, the finish time of task $i$ is forced to coincide with time point $p$.

$$
\begin{array}{ll}
t_{f}(i, p-1) \leq t(p)+H\left(1-W_{f}(i, p)\right) & \forall i \in I, p \in P, p>p_{1} \\
t_{f}(i, p-1) \geq t(p)-H\left(1-W_{f}(i, p)\right) & \forall i \in I_{z}, p \in P, p>p_{1}
\end{array}
$$

\section{Batch size constraints}

Batch size constraints impose limits on the amount of material that can be processed in a particular task. Constraints (19)-(24) impose minimum and maximum bounds on the batch size of tasks.

$$
\begin{gathered}
B_{s}(i, p) \geq B^{L}(i, p) W_{s}(i, p) \quad \forall i \in I, p \in P \\
B_{s}(i, p) \leq B^{U}(i, p) W_{s}(i, p) \quad \forall i \in I, p \in P \\
B_{f}(i, p) \geq B^{L}(i, p) W_{f}(i, p) \quad \forall i \in I, p \in P \\
B_{f}(i, p) \leq B^{U}(i, p) W_{f}(i, p) \quad \forall i \in I, p \in P \\
B_{p}(i, p) \geq B^{L}(i, p)\left(\sum_{p^{\prime} \in P}^{p^{\prime}<p} W_{s}\left(i, p^{\prime}\right)-\sum_{p^{\prime} \in P}^{p^{\prime} \leq p} W_{f}\left(i, p^{\prime}\right)\right) \quad \forall i \in I, p \in P \\
B_{p}(i, p) \leq B^{U}(i, p)\left(\sum_{p^{\prime} \in P}^{p^{\prime} \leq p} W_{s}\left(i, p^{\prime}\right)-\sum_{p^{\prime} \in P} W_{f}\left(i, p^{\prime}\right)\right) \quad \forall i \in I, p \in P
\end{gathered}
$$


Constraint (25) is a mass balance over two successive time points. Every task that has started at a previous time point can either continue being processed or finish at the following time point.

$$
B_{s}(i, p-1)+B_{p}(i, p-1)=B_{p}(i, p)+B_{f}(i, p) \quad \forall i \in I, p \in P, p>p_{1}
$$

The amount of a particular state $s$ consumed or produced by task $i$ at time point $p$ is calculated by constraints (26) and (27). The bounds on these variables are set by constraints (28) and (29).

$$
\begin{aligned}
& B_{I}(i, s, p)=\rho(i, s) B_{s}(i, p) \quad \forall i \in I, s \in S_{i}(i), p \in P \\
& B_{O}(i, s, p)=\rho(i, s) B_{f}(i, p) \quad \forall i \in I, s \in S_{o}(i), p \in P \\
& B_{I}(i, s, p) \leq B^{U}(i) \rho(i, s) W_{s}(i, p) \quad \forall i \in I, s \in S_{i}(i), p \in P \\
& B_{O}(i, s, p) \leq B^{U}(i) \rho(i, s) W_{f}(i, p) \quad \forall i \in I, s \in S_{o}(i), p \in P
\end{aligned}
$$

\section{Mass balance constraints}

The mass balance constraints ensure that the amount of material is conserved over each task. Constraints (30) and (31) ensure that the amount of state $s$ available at any time point $p$ can either continue being stored or be used by a task $i$. Furthermore, should state $s$ be produced from a task $i$, this state can also be used for further processing.

$$
\begin{aligned}
& S_{A}(s, p)+S_{S}(s, p)=S_{A}(s, p-1)+\sum_{i \in I_{o}(s)} B_{O}(i, s, p)-\sum_{i \in I_{i}(s)} B_{I}(i, s, p) \\
& \forall s \in S, p \in P, p>p_{1}
\end{aligned}
$$




$$
\begin{aligned}
& S_{A}(s, p)+S_{S}(s, p)=S_{A}^{0}(s)+\sum_{i \in I_{o}(s)} B_{O}(i, s, p)-\sum_{i \in I_{i}(s)} B_{I}(i, s, p) \\
& \forall s \in S, p \in P, p=p_{1}
\end{aligned}
$$

Constraint (32) imposes the upper bound of available material for each state $s$.

$$
S_{A}(s, p) \leq S_{A}^{U}(s) \quad \forall s \in S, p \in P
$$

\section{Tightening constraints}

The tightening constraints leads to improved solution times. Constraint (33) enforces that the sum of all task durations be less than or equal to the time horizon of interest.

$$
\sum_{i \in I_{j}(j)} \sum_{p \in P} \tau(i, p) \leq H \quad \forall j \in J
$$

Constraints (34) and (35) together enforce that the sum of task durations in a particular slot does not exceed the upcoming time point. In isolation, constraint (34) enforces the summation of the processing times of all tasks $i$ which start on a unit $j$ after $t(p)$ to be less than the total time remaining in the time horizon. Constraint (35) forces the durations of tasks which finish processing before time point $p$ to be less than or equal to the time associated with time point $p$, i.e. $t(p)$.

$$
\begin{gathered}
\sum_{i \in I_{j}(j)} \sum_{p \in P}^{p^{\prime} \geq p} \tau\left(i, p^{\prime}\right) \leq H-t(p) \quad \forall j \in J, p \in P \\
\sum_{i \in I_{j}(j)} \sum_{p^{\prime} \in P}^{p^{\prime} \leq p}\left(\alpha(i) W_{f}\left(i, p^{\prime}\right)+\beta(i) B_{f}\left(i, p^{\prime}\right)\right) \leq t(p) \quad \forall j \in J, p \in P
\end{gathered}
$$




\section{Appendix B}

\section{Linearisation constraints}

The model contains nonlinear constraints due to the multiplication of continuous variables. This makes the model difficult to solve. To provide the exact model with a good starting point, these constraints are linearised below as constraints (36)-(39). The linear model is then solved before the exact nonlinear model. The linearisation variables denoted by $\gamma$ are shown from constraints $(44)-(72)$.

$$
\begin{array}{ll}
E(n, p)=0.00085 \cdot 1.8 \sum_{i \in I}\left(\gamma_{5}(n, i, p)-C_{R}(n, i, p) T_{c t}(n)\right) & \\
Q_{u}(i, p)+c_{p}\left(\sum_{n \in N} \gamma_{4}(n, i, p)+\sum_{i^{\prime} \in I}^{i^{\prime} \neq i} \gamma_{2}\left(i^{\prime}, i, p\right)\right)=c_{p} \gamma_{3}(i, p) & \forall n \in P \\
\sum_{i \in I} \gamma_{1}(n, i, p)+\sum_{n^{\prime} \in N} R\left(n^{\prime}, n, p\right) T_{c t}\left(n^{\prime}\right) \leq T_{r e t}^{U}(n) \sum_{i \in I} C_{R}(n, i, p) & \\
\sum_{i \in I} \gamma_{4}(n, i, p)+\sum_{n^{\prime} \in N} \gamma_{6}\left(n^{\prime}, n, p\right)=M(n, p) T_{a m b} & \\
& \\
& \\
& \\
& \\
& \\
& \\
& \\
& \\
& \\
&
\end{array}
$$

Constraint (40) describes the approximated linearised profit. As can be seen, the operational costs have also been approximated to provide a good starting point for the exact nonlinear 
objective function.

$$
\begin{gathered}
\max \text { Profit }_{L}=\sum_{s \in S} \sum_{p \in P} \zeta(s) S S(s, p)-c_{T R}-c_{T C}-\frac{H}{H_{Y}} \sum_{p \in P} c_{P O}(p) \\
W_{r}\left(i^{\prime}, i, p\right) \leq \sum_{p^{\prime} \in P}^{p^{\prime} \leq p}\left(W_{s}\left(i, p^{\prime}\right)-W_{f}\left(i, p^{\prime}\right)\right) \quad \forall i, i^{\prime} \in I, p \in P, i^{\prime} \neq i \\
W_{r}\left(i^{\prime}, i, p\right) \leq \sum_{p^{\prime} \in P}^{p^{\prime} \leq p}\left(W_{s}\left(i^{\prime}, p^{\prime}\right)-W_{f}\left(i^{\prime}, p^{\prime}\right)\right) \quad \forall i, i^{\prime} \in I, p \in P, i^{\prime} \neq i \\
W_{r}\left(i^{\prime}, i, p\right)=W_{r}\left(i, i^{\prime}, p\right) \quad \forall i, i^{\prime} \in I, p \in P, i^{\prime} \neq i
\end{gathered}
$$

$\gamma_{1}(n, i, p)$ is the linearisation variable for the term $C_{R}(n, i, p) T_{\text {out }}(i)$. Constraints $(44)-(47)$ are used in defining this term.

$$
\begin{aligned}
& \gamma_{1}(n, i, p) \geq F_{\text {in }}^{U}(i) T_{\text {out }}(i, p)+C_{R}(n, i, p) T_{\text {out }}^{U}(i)-F_{\text {in }}^{U}(i) T_{\text {out }}^{U}(i) \\
& \forall n \in N, i \in I, p \in P \\
& \gamma_{1}(n, i, p) \leq F_{\text {in }}^{U}(i) T_{\text {out }}(i, p)+C_{R}(n, i, p) T_{\text {out }}^{L}-F_{\text {in }}^{U}(i) T_{\text {out }}^{L} \\
& \forall n \in N, i \in I, p \in P \\
& \gamma_{1}(n, i, p) \leq C_{R}(n, i, p) T_{\text {out }}^{U}(i) \quad \forall n \in N, i \in I, p \in P \\
& \gamma_{1}(n, i, p) \geq C_{R}(n, i, p) T_{\text {out }}^{L} \quad \forall n \in N, i \in I, p \in P
\end{aligned}
$$


$\gamma_{2}\left(i^{\prime}, i, p\right)$ is the linearisation variable for the term $F_{r}\left(i^{\prime}, i, p\right) T_{\text {out }}\left(i^{\prime}\right)$. Constraints $(48)-(51)$ are used in defining this term.

$$
\begin{aligned}
& \gamma_{2}\left(i^{\prime}, i, p\right) \geq F_{\text {in }}^{U}(i) T_{\text {out }}\left(i^{\prime}, p\right)+F_{r}\left(i^{\prime}, i, p\right) T_{\text {out }}^{U}\left(i^{\prime}\right)-F_{\text {in }}^{U}(i) T_{\text {out }}^{U}\left(i^{\prime}\right) \\
& \forall i, i^{\prime} \in I, p \in P \\
& \gamma_{2}\left(i^{\prime}, i, p\right) \leq F_{\text {in }}^{U}(i) T_{\text {out }}\left(i^{\prime}, p\right)+F_{r}\left(i^{\prime}, i, p\right) T_{\text {out }}^{L}-F_{\text {in }}^{U}(i) T_{\text {out }}^{L} \\
& \forall i, i^{\prime} \in I, p \in P \\
& \gamma_{2}\left(i^{\prime}, i, p\right) \leq F_{r}\left(i^{\prime}, i, p\right) T_{\text {out }}^{U}\left(i^{\prime}\right) \quad \forall i, i^{\prime} \in I, p \in P \\
& \gamma_{2}\left(i^{\prime}, i, p\right) \geq F_{r}\left(i^{\prime}, i, p\right) T_{\text {out }}^{L} \quad \forall i, i^{\prime} \in I, p \in P
\end{aligned}
$$

$\gamma_{3}(i, p)$ is the linearisation variable for the term $F_{\text {in }}(i, p) T_{\text {out }}(i)$. Constraints $(52)-(55)$ are used in defining this term.

$$
\begin{array}{cc}
\gamma_{3}(i, p) \geq F_{\text {in }}^{U}(i) T_{\text {out }}(i, p)+F_{\text {in }}(i, p) T_{\text {out }}^{U}(i)-F_{\text {in }}^{U}(i) T_{\text {out }}^{U}(i) & \\
\gamma_{3}(i, p) \geq F_{\text {in }}^{U}(i) T_{\text {out }}(i, p)+F_{\text {in }}(i, p) T_{\text {out }}^{L}-F_{\text {in }}^{U}(i) T_{\text {out }}^{L} & \forall i \in I, p \in P \\
\gamma_{3}(i, p) \leq F_{\text {in }}(i, p) T_{\text {out }}^{U}(i) & \forall i \in I, p \in P \\
\gamma_{3}(i, p) \geq F_{\text {in }}(i, p) T_{\text {out }}^{L} \quad \forall i \in I, p \in P &
\end{array}
$$


$\gamma_{4}(n, i, p)$ is the linearisation variable for the term $C_{S}(n, i, p) T_{\text {sup }}(n, p)$. Constraints $(56)-$ (59) are used in defining this term.

$$
\begin{array}{cc}
\gamma_{4}(n, i, p) \geq F_{i n}^{U}(i) T_{\text {sup }}(n, p)+C_{S}(n, i, p) T_{a m b}-F_{i n}^{U}(i) T_{a m b} & \\
\qquad n \in N, i \in I, p \in P & \\
\gamma_{4}(n, i, p) \leq F_{i n}^{U}(i) T_{\text {sup }}(n, p)+C_{S}(n, i, p) T_{c t}^{L}-F_{i n}^{U}(i) T_{c t}^{L} & \\
\quad \forall n \in N, i \in I, p \in P \\
\gamma_{4}(n, i, p) \leq C_{S}(n, i, p) T_{a m b} \quad \forall n \in N, i \in I, p \in P \\
\gamma_{4}(n, i, p) \geq C_{S}(n, i, p) T_{c t}^{L} \quad \forall n \in N, i \in I, p \in P
\end{array}
$$

where $T_{c t}^{L}$ is defined by equation (60).

$$
T_{c t}^{L}=\min _{n \in N} T_{c t}(n)
$$

$\gamma_{5}(n, i, p)$ is the linearisation variable for the term $C_{R}(n, i, p) T_{r e t}(n, p)$. Constraints (61)(64) are used in defining this term.

$$
\begin{aligned}
& \gamma_{5}(n, i, p) \geq F_{i n}^{U}(i) T_{r e t}(n, p)+C_{R}(n, i, p) T_{r e t}^{U}(n)-F_{\text {in }}^{U}(i) T_{\text {ret }}^{U}(n) \\
& \forall n \in N, i \in I, p \in P \\
& \gamma_{5}(n, i, p) \leq F_{i n}^{U}(i) T_{r e t}(n, p)+C_{R}(n, i, p) T_{c t}^{L}-F_{i n}^{U}(i) T_{c t}^{L} \\
& \forall n \in N, i \in I, p \in P \\
& \gamma_{5}(n, i, p) \leq C_{R}(n, i, p) T_{\text {ret }}^{U}(n) \quad \forall n \in N, i \in I, p \in P
\end{aligned}
$$




$$
\gamma_{5}(n, i, p) \geq C_{R}(n, i, p) T_{c t}^{L} \quad \forall n \in N, i \in I, p \in P
$$

$\gamma_{6}\left(n^{\prime}, n, p\right)$ is the linearisation variable for the term $R\left(n^{\prime}, n, p\right) T_{\text {sup }}(n, p)$. Constraints (65)(68) are used in defining this term.

$$
\begin{gathered}
\gamma_{6}\left(n^{\prime}, n, p\right) \geq O S^{U}(n) T_{\text {sup }}(n, p)+R\left(n^{\prime}, n, p\right) T_{a m b}-O S^{U}(n) T_{a m b} \\
\forall n, n^{\prime} \in N, p \in P \\
\gamma_{6}\left(n^{\prime}, n, p\right) \leq O S^{U}(n) T_{\text {sup }}(n, p)+R\left(n^{\prime}, n, p\right) T_{c t}^{L}-O S^{U}(n) T_{c t}^{L} \\
\quad \forall n, n^{\prime} \in N, p \in P \\
\gamma_{6}\left(n^{\prime}, n, p\right) \leq R\left(n^{\prime}, n, p\right) T_{a m b} \quad \forall n, n^{\prime} \in N, p \in P \\
\gamma_{6}\left(n^{\prime}, n, p\right) \geq R\left(n^{\prime}, n, p\right) T_{c t}^{L} \quad \forall n, n^{\prime} \in N, p \in P
\end{gathered}
$$

$\gamma_{7}\left(n^{\prime}, n, p\right)$ is the linearisation variable for the term $R\left(n^{\prime}, n, p\right) T_{r e t}(n, p)$. Constraints (69)(72) are used in defining this term.

$$
\begin{aligned}
& \gamma_{7}\left(n^{\prime}, n, p\right) \geq O S^{U}(n) T_{\text {ret }}(n, p)+R\left(n^{\prime}, n, p\right) T_{\text {ret }}^{U}(n)-O S^{U}(n) T_{\text {ret }}^{U}(n) \\
& \forall n, n^{\prime} \in N, p \in P
\end{aligned}
$$

$$
\begin{array}{cc}
\gamma_{7}\left(n^{\prime}, n, p\right) \leq O S^{U}(n) T_{r e t}(n, p)+R\left(n^{\prime}, n, p\right) T_{c t}^{L}-O S^{U}(n) T_{c t}^{L} & \\
& \forall n, n^{\prime} \in N, p \in P \\
& \\
\gamma_{7}\left(n^{\prime}, n, p\right) \leq R\left(n^{\prime}, n, p\right) T_{r e t}^{U}(n) & \forall n, n^{\prime} \in N, p \in P \\
\gamma_{7}\left(n^{\prime}, n, p\right) \geq R\left(n^{\prime}, n, p\right) T_{c t}^{L}(n) \quad & \forall n, n^{\prime} \in N, p \in P
\end{array}
$$

